\title{
Elevated Choline-Containing Compound Levels in Rapid Cycling Bipolar Disorder
}

\author{
Bo Cao*,', Jeffrey A Stanley', Ives Cavalcante Passos ${ }^{3}$, Benson Mwangi', Sudhakar Selvaraj', \\ Giovana B Zunta-Soares' and Jair C Soares'
}

'Department of Psychiatry and Behavioral Sciences, The University of Texas Health Science Center at Houston, TX, USA; ${ }^{2}$ Department of Psychiatry \& Behavioral Neurosciences, School of Medicine, Wayne State University, Detroit, MI, USA; ${ }^{3}$ Graduation Program in Psychiatry and Department of Psychiatry, Universidade Federal do Rio Grande do Sul (UFRGS), Porto Alegre (RS), Brazil

\begin{abstract}
Previous studies have found increased levels of choline-containing compounds (ie, glycerophosphocholine plus phosphocholine $(\mathrm{GPC}+\mathrm{PC})$ ) in bipolar disorder using in vivo proton magnetic resonance spectroscopy ('H MRS), especially in bipolar I disorder (BD-I). Increased levels of GPC+PC suggest alterations in the membrane phospholipids metabolism in bipolar disorder. Rapid cycling (RC) bipolar disorder is considered as a severe course of bipolar disorder, but it is unclear whether rapid cycling bipolar disorder is linked to highly altered membrane phospholipid metabolism. The purpose of this study was to investigate whether the regional extent of elevated GPC+PC were greater in BD-I patients with rapid cycling compared to BD-I patients without rapid cycling and healthy controls. Using a multi-voxel 'H MRS approach at 3 Tesla with high spatial resolution and absolute quantification, GPC+PC levels from the anterior cingulate cortex (ACC), caudate and putamen of 16 RC BD-I, 34 non-RC BD-I and 44 healthy controls were assessed. We found significantly elevated GPC+PC levels in ACC, putamen and caudate of RC BD-I patients compared to healthy controls $(P<0.005)$ and in ACC compared to non-RC BD-I patients $(P<0.05)$. These results suggest greater alteration of membrane phospholipid metabolisms in rapid cycling BD-I compared to non-rapid-cycling BD-I. Neuropsychopharmacology (2017) 42, 2252-2258; doi:I 0.I 038/npp.20 I7.39; published online 29 March 2017
\end{abstract}

\section{INTRODUCTION}

In the United States, approximately six million people suffer from bipolar disorder (BD) (Lee et al, 2010). According to the World Health Organization (WHO), bipolar disorder is among the 10 leading causes of disability-adjusted life years in young adults (Mathers and Loncar, 2006). The rates of completed suicide among patients with bipolar disorder are $7.8 \%$ in men and $4.8 \%$ in women, which are more than 10 times higher than the non-psychiatric population (Nordentoft et al, 2011). Interestingly, about one-third of bipolar disorder patients are identified as having rapid cycling (RC) of mood episodes (Lee et al, 2010), which is viewed as a more severe course of bipolar disorder (Kupka et al, 2005). According to DSM-V (and DSM-IV), at least four mood episodes over a 12-month period must be present for a patient to be diagnosed as the rapid cycling course of bipolar disorder. Rapid cycling bipolar disorder patients can experience seven times of the mean number of manic and hypomanic episodes and twice of the mean number of depressive episodes as the non-RC bipolar disorder patients (Kupka et al, 2005). As a result, rapid cycling bipolar

* Correspondence: Dr B Cao, Department of Psychiatry and Behavioral Sciences, The University of Texas Health Science Center at Houston, 194| East Road, Houston, TX 77054, USA, Tel: +I 7I3 4862624 , Fax: + 713486 2553, E-mail: CloudBoCao@gmail.com

Received 4 November 2016; revised 30 January 2017; accepted 4 February 2017; accepted article preview online 21 February 2017 disorder patients have been reported up to 132 days of being functionally impaired from day-to-day activities due to mood episodes within a 12-month period, compared to 85 days for non-RC BD (Lee et al, 2010). Rapid cycling is also persistent, as more than $50 \%$ of rapid cycling bipolar disorder patients continue to suffer beyond 10 years (Koukopoulos et al, 2003). Moreover, current pharmacological treatments of bipolar disorder, such as lithium and carbamazepine, are generally less effective for patients with rapid cycling (Post et al, 2012). This has led to fewer treatment options for rapid cycling bipolar disorder patients (Fountoulakis et al, 2013). Collectively, the abovementioned suggests that the severity of rapid cycling in bipolar disorder is much greater; however, the neuropathology of rapid cycling is poorly understood, especially in the patients with bipolar I disorder (BD-I). One neuroimaging study has reported a smaller ventral prefrontal cortex volume in RC BD-I patients than non-RC BD-I patients (Blumberg et al, 2006). Little is known with respect to alterations in the biochemistry of RC BD-I patients.

The basal ganglia and anterior cingulate cortex (ACC) are critical brain regions associated with reward processing and mood regulation (Bush et al, 2000; Sesack and Grace, 2010). Previous studies have found increased levels of cholinecontaining compounds, (ie, glycerophosphocholine plus phosphocholine $(\mathrm{GPC}+\mathrm{PC})$ ) in these regions of bipolar disorder patients using in vivo proton magnetic resonance spectroscopy ( $\left({ }^{1} \mathrm{H}\right.$ MRS) (Cao et al, 2016a; Dager et al, 2004; 
Hamakawa et al, 1998; Kato et al, 1996; Moore et al, 2000), especially in patients with bipolar I disorder (BD-I) (Cao et al, 2016b; Moore et al, 2000). GPC and PC are catabolic and anabolic metabolites of cell membrane phospholipids (MPLs), respectively (McClure et al, 1994), and increased GPC+PC suggests altered MPL metabolism that may be associated with bipolar disorder. Rapid cycling bipolar disorder is considered as a severe course of bipolar disorder due to its high frequency of mood episode alternations, which could be linked to a greater degree in altered MPL metabolism evidenced by increased GPC+PC, driven by a shift of greater catabolic activity over anabolic activity in the metabolism of MPLs compared to non-RC bipolar disorder. Therefore, the purpose of this study was to investigate whether the regional extent of elevated GPC $+\mathrm{PC}$ was greater in BD-I patients with rapid cycling compared to patients without rapid cycling and healthy controls using a multi-voxel ${ }^{1} \mathrm{H}$ MRS approach with high spatial resolution and with absolute quantification. We hypothesized that the extent of increased GPC+PC levels would be greater in RC BD-I patients compared to non-RC $\mathrm{BD}-\mathrm{I}$ and healthy subjects.

\section{MATERIALS AND METHODS}

\section{Participants}

Ninety-four adults between the ages of 18 and 65 years old participated in the study, which included 16 RC BD-I and 34 non-RC BD-I patients and 44 healthy controls (HC) (Table 1). Bipolar disorder patients were recruited from outpatient clinics at the University of North Carolina at Chapel Hill and HC were recruited through local flyers and media advertisements. All patients met the DSM-IV-R criteria for bipolar I disorder using the Structured Clinical Interview for the Diagnostic and Statistical Manual of Mental Disorders Axis I (SCID-I), which was administered by an independent psychiatrist or trained research psychologist. The rapid cycling criteria was met if a patient experienced in the past at least four mood episodes over a 12-month period. Mood symptoms were assessed using the Young Mania Rating Scale (YMRS) (Young et al, 1978) and Hamilton Rating Scale for Depression (HAM-D)(Hamilton, 1980). Any participant with a history of chronic medical issues including cardiovascular and neurological disorders was excluded. HC with a history of any Axis I disorder themselves or in their first-degree relatives or use of psychoactive medication less than 2 weeks before the study were excluded. All participants showed a negative urine drug screen, and all female participants showed a negative urine pregnancy test on the scan day. The study protocol was approved by the local Institutional Review Board. Informed consent was obtained from all the participants. Participants' information of demographics, current mood states and medications are summarized in Table 1.

\section{In vivo ${ }^{1} \mathrm{H}$ MRS Acquisition}

The MRI and ${ }^{1} \mathrm{H}$ MRS data were acquired on a 3-Tesla Siemens Magnetom Allegra syngo MR scanner (Siemens Medical Solutions, Erlangen, Germany). Regarding the protocol, scout MRI images were first acquired followed by
Table I Demographic Information of Subjects

\begin{tabular}{|c|c|c|c|c|c|}
\hline & HC $(n=44)$ & $\begin{array}{c}\text { non-RC } \\
\text { BD-I } \\
(n=34)\end{array}$ & $\begin{array}{c}\text { RC BD-I } \\
(n=16)\end{array}$ & $F / X^{2}$ & $P$-value \\
\hline Age (years) & $35.4 \pm 13.4$ & $36.8 \pm 13.7$ & $33.5 \pm 8.4$ & 0.37 & 0.69 \\
\hline Gender & $20 M+24 F$ & $12 \mathrm{M}+22 \mathrm{~F}$ & $9 M+7 F$ & 2.01 & 0.36 \\
\hline Education (years) & $16.6 \pm 3.8$ & $14.2 \pm 2.4$ & $14.5 \pm 2.4$ & 5.97 & 0.004 \\
\hline YMRS & $0.4 \pm 0.7$ & $5.0 \pm 5.7$ & $5.4 \pm 4.3$ & $|6.8|$ & $<0.00$ I \\
\hline HAM-D & $0.4 \pm 0.9$ & $12.8 \pm 8.7$ & $13.5 \pm 10.1$ & 39.41 & $<0.001$ \\
\hline Duration as BD-I & - & $8.7 \pm 8.7$ & $6.6 \pm 5.6$ & 0.69 & 0.41 \\
\hline $\begin{array}{l}\text { Duration as any } \\
\text { mood disorder }\end{array}$ & - & $15.2 \pm \mid 1.1$ & $11.4 \pm 10.8$ & 1.10 & 0.30 \\
\hline Current mood & & & & $1.164^{\mathrm{a}}$ & $0.762^{\mathrm{a}}$ \\
\hline Euthymic & - & 8 (23.5\%) & $4(25.0 \%)$ & & \\
\hline Depressed & - & 21 (61.8\%) & $8(50.0 \%)$ & & \\
\hline Manic & - & | (2.9\%) & | (6.3\%) & & \\
\hline Mixed & - & $2(5.9 \%)$ & $2(12.5 \%)$ & & \\
\hline Missing & & $2(5.9 \%)$ & I (6.3\%) & & \\
\hline \multicolumn{6}{|l|}{ Current medication } \\
\hline Lithium & - & $3(8.8 \%)$ & $2(12.5 \%)$ & 0.279 & 0.597 \\
\hline Antidepressant & - & $6(17.6 \%)$ & $3(18.8 \%)$ & 0.067 & 0.796 \\
\hline Anticonvulsant & - & 7 (20.6\%) & $4(25.0 \%)$ & 0.297 & 0.586 \\
\hline Benzodiazepines & - & $6(17.6 \%)$ & $4(25.0 \%)$ & 0.633 & 0.426 \\
\hline $\begin{array}{l}\text { Not on } \\
\text { medication }\end{array}$ & - & 21 (61.8\%) & $8(50.0 \%)$ & 0.175 & 0.675 \\
\hline
\end{tabular}

Abbreviations: $\mathrm{BD}$, bipolar disorder; $\mathrm{HC}$, healthy control; $\mathrm{RC}$, rapid cycling; HAM-D, Hamilton Rating Scale for Depression; YMRS, Young Mania Rating Scale. ${ }^{a} \chi^{2}$-test for the distribution of mood status between non-RC and RC BD-I patients.

a set of $\mathrm{T}_{1}$-weighted MRI images using the magnetization prepared rapid acquisition gradient-echo (MPRAGE) sequence $\left(\mathrm{TR}=1750 \mathrm{~ms}, \mathrm{TE}=4.38 \mathrm{~ms}\right.$, flip angle $=8^{\circ}$, field of view $(\mathrm{FOV})=256 \times 256 \mathrm{~mm}^{2}$, number of axial slices $=160$, slice thickness $=1 \mathrm{~mm}$, number of excitations $=1$, matrix size $=256 \times 208$ ). Two separate ${ }^{1} \mathrm{H}$ MRS measurements were acquired using a three-dimensional (3D) multi-voxel acquisition scheme, which combined the point resolved spectroscopy (PRESS) sequence with the chemical shift imaging (CSI) sequence. The first was acquired by placing the PRESS volume of interest (VOI) in the frontal lobe $\left(70 \times 50 \times 40 \mathrm{~mm}^{3}\right)$, which included the anterior portion of the ACC and the second in the basal ganglia area $\left(100 \times 65 \times 60 \mathrm{~mm}^{3}\right)$. The acquisition parameters included the following: $\mathrm{TR}=1410 \mathrm{msec}, \mathrm{TE}=80 \mathrm{~ms}$ (Schubert et al, 2004), $\mathrm{FOV}=160 \times 160 \times 80 \mathrm{~mm}^{3}$, acquired CSI matrix $=14$ $\times 14 \times 8$ and zero-filled to $16 \times 16 \times 8$, nominal pixel dimension $=10 \times 10 \times 10 \mathrm{~mm}^{3}$, complex spectral data points $=2048$, spectral bandwidth $=2.0 \mathrm{kHz}$, water suppression using CHESS pulses (Haase et al, 1985) and number of averages $=1$. Additionally, unsuppressed water measurements for absolute quantification were acquired using the identical parameters except the acquired CSI matrix was $8 \times 8 \times 8$ and zero-filled to $16 \times 16 \times 8$. The locations of the voxels are shown in Figure 1. 


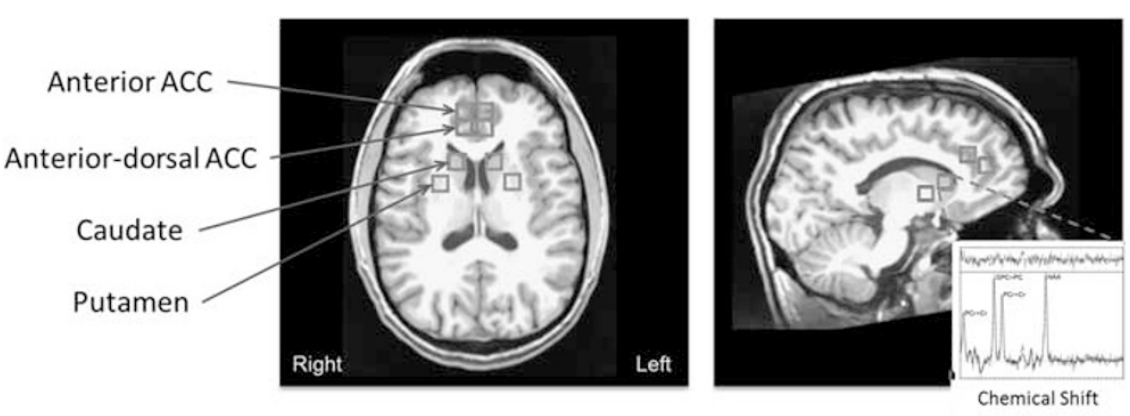

Figure I Voxel locations of MRS acquisition. A sample fitted spectrum acquired from the left caudate is shown in the insert plot. The peaks of the spectrum from the right to the left indicate components for NAA, PCr+Cr. GPC+PC and PCr+Cr, respectively. The top part of the insert plot shows the residuals of the fitting. MRS, magnetic resonance spectroscopy.

\section{Data Processing}

The post-processing included extracting and quantifying the ${ }^{1} \mathrm{H}$ MRS signal from four different left and right voxel locations and estimating the tissue fraction within those extracted ${ }^{1} \mathrm{H}$ MRS voxels (ie, the percent gray and white matter and cerebrospinal fluid (CSF)). The procedure from the beginning to the end was $100 \%$ automated and independent of operator inputs (Wu et al, 2014). The voxel locations of interest, which included the anterior ACC, anterior dorsal parts of ACC, caudate head, and putamen, were predefined anatomically on an MNI template brain image, and the voxels were mapped to the subject space using the co-registration transformation between the $\mathrm{T}_{1}$-weighted images and the template image. The coordinates of the voxel location in subject space were then used to shift the $3 \mathrm{D}$ multi-voxel grid to ensure a voxel was centered at that location and then the ${ }^{1} \mathrm{H}$ MRS signal of that voxel were extracted for quantification. This innovative procedure consistently and systematically placed the voxels in the specified anatomical locations between subjects, and has been demonstrated to be accurate and reliable (Wu et al, 2014). These voxel locations were also mapped on the tissuesegmented images, in which the tissue fraction within each voxel was estimated. In brief, the $\mathrm{T}_{1}$-weighted images were corrected for any $B_{1}$ field bias, the brain was extracted and images segmented into partial volume maps of gray and white matter tissue, and CSF/extra-cortical space using FreeSurfer and FSL tools (eg, FLIRT, NU_CORRECT, BET and FAST)(Dale et al, 1999; Smith et al, 2004).

GPC+PC levels were quantified using the Linear Combination (LC) Model software with a simulated basis set for the a priori knowledge (Provencher, 2001), along with other metabolites, such as $\mathrm{N}$-acetylaspartate (NAA), phosphocreatine plus creatine $(\mathrm{PCr}+\mathrm{Cr})$, glutamate $(\mathrm{Glu})$, myo-inositol (myo-Ins) L-alanine, aspartate, $\gamma$-aminobutyric acid, glucose, glutathione, L-lactate, $\mathrm{N}$-acetylaspartylglutamate, scyllo-inositol and taurine as well as lipid resonances and macromolecule resonances (Seeger et al, 2003). We focused on the GPC+PC levels, but other metabolites with adequate quantification reliability were also reported (see Supplementary Materials), such as NAA, PCr+Cr, myo-Ins and Glu (ie, Cramer-Rao lower bound (CRLB) values $<25 \%$ ). The following inclusion criteria were applied to the fitted spectrum of each voxel: the signal-to-noise ratio equal to or greater than 5, CRLB values equal to of $<25 \%$, absolute data shift $\leqslant 0.1$ p.p.m., and absolute first-order phase correction equal to or less than $30 \%$ p.p.m. The unsuppressed water signal along with the appropriate correction factors related to the proportions of gray matter, white matter and CSF within the voxel were applied to obtain absolute quantification values with institutional units (IU) (Kreis et al, 1993).

\section{Statistical Analysis}

For each voxel location, a group-level difference of GPC+PC levels between RC BD-I, non-RC BD-I, and $\mathrm{HC}$ was estimated using the general linear model (GLM) with age and gender as co-variates. GPC+PC levels from the left and right voxels of the same brain region were averaged before entered into the GLM, because pilot analysis did not find any confounding effect from laterality on the diagnosis effect on the metabolite levels (see Supplementary Materials for the laterality main effect alone). The post-hoc analyses within the three groups were based on the mean values adjusted for age and gender, and the $P$-values were Bonferroni corrected for the three group comparisons.

To investigate whether the alteration of GPC+PC was due to the current rapid cycling or was a general marker for both current rapid cycling and retrospective rapid cycling, further post-hoc tests were performed by separating the currently non-RC patients into two groups: patients probable to have a history of rapid cycling and patients not probable to have a history of rapid cycling. The GPC+PC levels of these two groups were compared to the patients with RC BD-I. Whether the patients were probable to have a history of rapid cycling was determined by the average number of mood episodes per year, which was calculated by dividing the total number of mood episodes (manic, depressive and mixed) by the duration since the first diagnosis with any mood disorder. If the average number of mood episodes per year was greater than 3 , the patient should experience four or more mood episodes within a certain 12-month period. Thus, for this further post-hoc analysis, we consider that within the non-RC patients, those with an average number of mood episodes per year greater than 3 as the non-RC patients with a probable rapid cycling history, and the patients with an average number of mood episodes per year $\leqslant 3$ as the non-RC patients probably without a probable rapid cycling history. In all the analyses, $P$-values smaller than 0.05 were considered as significant. The statistical analysis was conducted with SPSS version 23 (IBM Corp., Armonk, NY). 


\section{RESULTS}

Age and gender were not distributed differently neither across the three groups nor between the $\mathrm{RC}$ and non-RC BDI patients $(P>0.05)$. There were significant differences in education, YMRS and HAM-D across the three groups, however, none of which were significantly different between $\mathrm{RC}$ and non-RC BD-I patients $(P>0.05)$. None of the mood states or medications showed significantly different distributions between the $\mathrm{RC}$ and non-RC groups according to $\chi^{2}$-tests $(P>0.05)$ (Table 1). The illness duration with any mood disorder was not significantly different between the $\mathrm{RC}$ and non-RC BD-I patients $(P>0.05)$.

For GPC+PC, the main effect of the diagnosis group term was significant in the anterior $\operatorname{ACC}[\mathrm{F}(2,70)=5.532$; $P=0.006]$, anterior dorsal $\mathrm{ACC}[\mathrm{F}(2,74)=6.792 ; P=0.002]$, caudate $[\mathrm{F}(2,44)=5.726 ; P=0.006]$ and putamen $[\mathrm{F}(2$, $44)=7.147 ; P=0.002]$ (Supplementary Table S1). With posthoc comparisons, RC BD-I patients showed consistently elevated GPC+PC levels in these four regions compared to HC $(P$-values $<0.005)$, and in both ACC regions compared to non-RC BD-I patients $(P$-values $<0.05) \quad$ (Figure 2 and Supplementary Table S1).

We performed preliminary comparisons between RC BD-I patients and the currently non-RC BD-I patients with or without a probable history of rapid cycling. We found that the GPC+PC levels were significantly higher in currently RC BD-I patients compared to non-RC BD-I patients without a probable history of rapid cycling in the anterior ACC $(t=2.390 ; P=0.022)$ and anterior dorsal ACC $(t=2.715$; $P=0.010$ ), and marginally higher in currently RC BD-I patients compared to non-RC BD-I patients with a probable history of rapid cycling in the anterior ACC $(t=1.949$; $P=0.059)$ anterior dorsal ACC $(t=1.873 ; P=0.068)$, and putamen $(t=1.966 ; P=0.062)$. The YMRS and HAMD were not significantly different between these three groups $(P>0.05)$, meaning that the GPC+PC differences that we found were unlikely due to symptom severity difference.

The main effect of the diagnosis group term for NAA was also significant in the anterior $\operatorname{ACC}[\mathrm{F}(2,70)=3.620$;

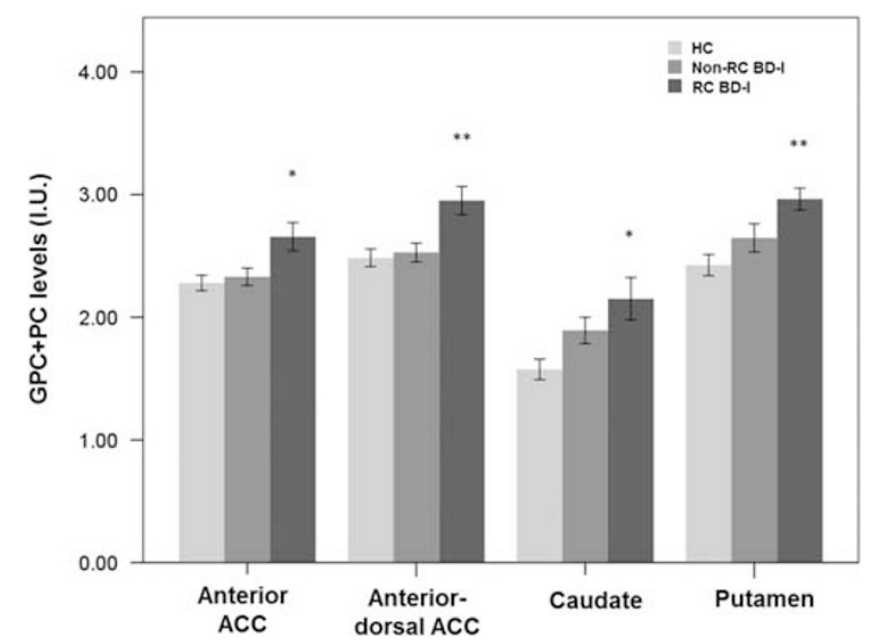

Figure 2 Elevated GPC+PC in anterior and anterior dorsal part of ACC, caudate and putamen in RC BD-I patients, compared to $\mathrm{HC}$ and non-RC BD-I. $* P<0.05$; $* * P<0.005$. BD, bipolar; GPC+PC, glycerophosphocholine plus phosphocholine; $\mathrm{HC}$, healthy control; RC, rapid cycling.
$P=0.032]$ and anterior dorsal ACC $[\mathrm{F}(2,74)=4.030$; $P=0.022]$, but not in the caudate or putamen $(P>0.05)$. Post-hoc results showed higher NAA levels in these two ACC regions of RC BD-I patients compared to non-RC BD-I $(P$-values $<0.05)$ and HC $(P$-values $\leqslant 0.055)$. Other metabolites, such as $\mathrm{PCr}+\mathrm{Cr}$, myo-Ins and Glu, did not show an effect on the diagnosis group term ( $P$-values $>0.05)$. No group differences were found for signal-to-noise ratio or Cramer-Rao lower bounds ( $P$-values $>0.05)$.

\section{DISCUSSION}

To our knowledge, this is the first study to investigate the biochemical alterations related to rapid cycling in patients with BD-I using a multi-voxel ${ }^{1} \mathrm{H}$ MRS approach with high spatial resolution and with absolute quantification. We found significantly higher GPC+PC levels in key areas related to mood processing, such as ACC, caudate and putamen in RC BD-I patients compared to healthy subjects. These findings are consistent with the previous findings of elevated GPC $+\mathrm{PC}$ levels in ACC and basal ganglia in bipolar disorder (Dager et al, 2004; Hamakawa et al, 1998; Kato et al, 1996; Moore et al, 2000), but provide further evidence that the pathology that causes or accompanies the elevated GPC+PC levels are probably more severe in RC BD-I patients than non-RC BD-I patients. Although non-RC BD-I patients also showed a non-significant increase of GPC+PC levels in caudate and putamen compared to $\mathrm{HC}$, but the increase was most significant in RC BD-I patients. With a preliminary analysis, we also found that the elevated GPC+PC levels were most likely due to the current rapid cycling, but not the retrospective rapid cycling history.

GPC and PC are associated with the metabolism of cell membrane phospholipids (MPLs). PC is a precursor and GPC a breakdown product of MPLs, and PC is also a precursor and breakdown product of sphingomyelin. In tissues, MPLs naturally form membrane bilayers that physically separate the intracellular components from the extracellular environment of neurons, astrocytes (oligodendrocytes) and microglia (McClure et al, 1994). The steadystate MPL turnover rate reflecting the balance between synthesis and breakdown of MPLs varies highly with a shift of greater synthesis activity during brain development to an imbalance of greater MPL breakdown during aging (Stanley, 2002; Stanley et al, 2000). The dynamics are reflected in the varying demand of active MPL synthesis during development of cell membrane components required in dendritic and synaptic connections (ie, neuropil expansion). This is followed by decreases in precursor levels and increases in breakdown products coinciding with maturation (ie, pruning or neuropil contraction) while the shift of continued increasing breakdown products reflecting greater neuropil contraction have been observed in normal aging. Interestingly, increased GPC+PC levels have also been reported in Alzheimer's Disease and multiple sclerosis compared to healthy controls (Kantarci et al, 2003; Mader et al, 2000). Thus, although ${ }^{1} \mathrm{H}$ MRS cannot differentiate between the implications of synthesis $v s$ degradation of MPL, the increased GPC+PC observed in rapid cycling may suggest a greater shift in the degradation activity of MPL leading to a contraction in the neuropil. 
Higher than normal levels of $\mathrm{GPC}+\mathrm{PC}$ in caudate were also found in major depression and schizophrenia (Bustillo et al, 2002; Gabbay et al, 2007), which were associated to the abnormal metabolisms of MPLs (Pettegrew et al, 1991). Interestingly, in patients with schizophrenia, glucose metabolism measured by $\left[{ }^{18} \mathrm{~F}\right]$ fluorodeoxyglucose (FDG) positron emission tomography (PET) was found to be low in caudate (Buchsbaum et al, 1992), which may indicate energy metabolism deficit probably due to mitochondrial dysfunction (Stork and Renshaw, 2005). Elevated GPC+PC levels were also observed in inflammatory demyelination diseases (ie, multiple sclerosis (Mader et al, 2000)) and brain inflammatory responses triggered by other infective diseases (ie, hepatitis $\mathrm{C}$ virus (HCV) and human immunodeficiency virus (HIV) (Bokemeyer et al, 2011; Chang et al, 2013)). Emerging evidence from clinical and preclinical studies suggest dysfunctional immune regulation in bipolar disorder (Fillman et al, 2014; Najjar et al, 2013; Rao et al, 2010; Stertz et al, 2013; Watkins et al, 2014). Our findings are in line with the theories of mitochondrial dysfunction and neuroinflammation in bipolar disorder, and indicate that rapid cycling may be related to more severe dysfunctions of mitochondria and immune dysregulation compared to non-RC bipolar disorder, which is also in line with the neuroprogression theory of bipolar disorder (Cao et al, 2016a,b, 2017; Lavagnino et al, 2015; Mwangi et al, 2016; Post et al, 2012).

As ACC and striatum are associated with mood processing (Phillips et al, 2008), the abnormal MPL metabolisms in these regions may be related to the pathology and emergence of the mood episodes. As a severe course of bipolar disorder, rapid cycling bipolar disorder manifests with more waxes and wanes of mood states and polarity switches of mood episodes. It is intuitive for us to hypothesis that rapid cycling correlates with significant biochemical changes in ACC and basal ganglia regions compared to non-RC BD-I and healthy subjects, which is hereby supported by our findings of elevated GPC+PC levels in the anterior and dorsal-anterior ACC, caudate and putamen in RC BD-I patients.

The increased GPC+PC levels in the anterior and anterior dorsal ACC of currently RC BD-I patients compared to nonRC BD-I patients with and without a probable history of rapid cycling may indicate that the elevated $\mathrm{GPC}+\mathrm{PC}$ was probably due to the current rapid cycling, but not the retrospective rapid cycling history. Although the results are preliminary, these observations indicate that it may be during the course of rapid cycling that the altered MPL metabolism associated with bipolar disorder are most significant, and elevated GPC+PC could be a potential marker for rapid cycling.

Although some studies linked the imbalance of cholinergic systems with bipolar disorders (van Enkhuizen et al, 2014; Janowsky et al, 1972; Tamas et al, 2003; Young and Dulcis, 2015), it is unlikely that the observed elevated GPC+PC could directly support this interpretation in rapid cycling due to the small contribution of choline and acetylcholine in the ${ }^{1} \mathrm{H}$ MRS signals. Studies have shown that a cholinergic agonist did alter the GPC+PC signals observed by ${ }^{1} \mathrm{H}$ MRS in the patients diagnosed with the Alzheimer's disease (Frederick et al, 2002; Satlin et al, 1997). Further studies are necessary to investigate whether the elevated GPC+PC in rapid cycling could be indirectly linked to the altered cholinergic activity.
Our findings of simultaneous higher $\mathrm{GPC}+\mathrm{PC}$ and NAA levels in ACC regions of RC BD-I patients than non-RC BD-I patients and healthy controls were consistent with a previous study investigating the left dorsolateral prefrontal cortex in a small sample of rapid cycling type two bipolar (BD-II) patients and controls (Michael et al, 2009). The authors found that NAA, GPC+PC, PCr+Cr and Glu+Gln levels were all higher in RC BD-II patients after correcting for CSF and gray matter fraction. However, in our study, $\mathrm{PCr}+\mathrm{Cr}$ and $\mathrm{Glu}$ + Gln levels, as well as myo-Ins levels, tended to be higher in RC BD-I patients than controls and non-RC BD-I patients, although they did not reach the significant level of 0.05 . The elevated absolute NAA levels in cortical regions of rapid cycling bipolar disorder patients may indicate that the neuronal viability was not decreased as commonly believed for bipolar disorder. There is evidence of increased NAA in rare demyelination diseases, such as the Canavan's disease that is caused by an overproduction of the enzyme aspartoacylase that produces excess NAA (Grodd et al, 1990). Interestingly, lithium, a well-known mood stabilizer, seems to normalize the NAA levels in the Canavan's disease and bipolar disorder (Assadi et al, 2010). In general NAA levels are known to reflect the functioning neuroaxonal tissue including the maintenance of myelin due to its presence in oligodendrocytes (Baslow, 2003; Chakraborty et al, 2001; Stanley et al, 2007). To further complicate the interpretation of NAA, some recent studies have shown decreased NAA in the occipital cortex in response to visual stimulation (Baslow et al, 2016; Landim et al, 2016), suggesting that the dynamics of NAA may be related to neuronal activity. Thus, the increased NAA observed in our study may be associated with a general reduction of neuronal activity in the ACC that is unique to the RC BD patients, which is speculative and warrants further investigations.

The current study has several limitations. The definition of rapid cycling using four or more mood episodes within a 12-month period is still open to debate (Kupka et al, 2005). However, there have been evidence showing the validity and reliability of such a definition (Koukopoulos et al, 2003; Post et al, 2003, 2012). The GPC+PC levels detected by ${ }^{1} \mathrm{H}$ MRS could not differentiate GPC and PC, and thus could not provide the precise insight on the relationship between MPL synthesis and degradation related to rapid cycling. Future studies using phosphorus MRS with proton decoupling may help to disentangle the direction of altered metabolism in MPLs. The sample size of our study was still not sufficiently large to account every aspect related to rapid cycling bipolar disorder. Further, because the volume of interest has to be large and sufficiently away from the scalp, we could not cover some brain regions, such as the dorsal lateral prefrontal cortex, unlike some single voxel studies that could optimize the angle and location of the voxel for frontal regions. The preliminary findings between the current RC BD-I patients and non-RC BD-I patients with a probable rapid cycling history were based on a limited sample size, and grouping the non-RC BD-I patients into those with and without a probable rapid cycling history was based on the self-reports of the number of mood episodes and could be possibly inaccurate for some of the patients. Thus, these findings need to be interpreted with caution. Moreover, as a cross-sectional study, the causal relationship between rapid cycling and the observed biochemical alterations could not be determined. 
To investigate whether the biochemical alterations, especially elevated GPC+PC levels, cause rapid cycling or they are just an accompanied effect of rapid cycling, we need to use a large sample followed up longitudinally before and through the whole rapid cycling course of bipolar disorder.

In summary, we found consistently elevated absolute GPC + PC levels in the anterior and anterior dorsal ACC, caudate and putamen in RC BD-I patients compared to healthy subjects. According to a further preliminary analysis, the elevated GPC+PC levels were likely due to the current rapid cycling, but not the retrospective rapid cycling history. To our knowledge, this is the first study investigating the biochemical alterations related to rapid cycling in BD-I patients using a multi-voxel ${ }^{1} \mathrm{H}$ MRS approach with high spatial resolution and with absolute quantification. The elevated GPC+PC in ACC, caudate and putamen may reflect significantly altered metabolism of MPL in these regions in rapid cycling compared to non-RC BD-I and healthy subjects. These findings may help us understand the brain pathology of the rapid cycling mood episodes in bipolar patients, and may lead to unique insights to understand how manic and depressive episodes emerge due to biochemical alterations caused by external and internal stressful events in the future.

\section{FUNDING AND DISCLOSURE}

BC, JAS, ICP, BM, SS, and GBZ-S reported no biomedical financial interests or potential conflicts of interest. GBZ-S has received grants/research support from Forrest, BMS, J\&J, Merck, Stanley Medical Research Institute, and has been a speaker for Pfizer and Abbott.

\section{ACKNOWLEDGMENTS}

Supported in part by NIMH grant R01 085667, the Dunn Research Foundation, and the Pat Rutherford, Jr. Endowed Chair in Psychiatry (Jair C. Soares) and NARSAD Young Investigator Grants of The Brain \& Behavior Research Foundation (Bo Cao).

\section{REFERENCES}

Assadi M, Janson C, Wang DJ, Goldfarb O, Suri N, Bilaniuk L et al (2010). Lithium citrate reduces excessive intra-cerebral N-acetyl aspartate in Canavan disease. Eur J Paediatr Neurol 14: 354-359.

Baslow MH (2003). N-acetylaspartate in the vertebrate brain: metabolism and function. Neurochem Res 28: 941-953.

Baslow MH, Cain CK, Sears R, Wilson DA, Bachman A, Gerum S et al (2016). Stimulation-induced transient changes in neuronal activity, blood flow and $\mathrm{N}$-acetylaspartate content in rat prefrontal cortex: a chemogenetic fMRS-BOLD study. NMR Biomed 29: 1678-1687.

Blumberg HP, Krystal JH, Bansal R, Martin A, Dziura J, Durkin K et al (2006). Age, rapid-cycling, and pharmacotherapy effects on ventral prefrontal cortex in bipolar disorder: a crosssectional study. Biol Psychiatry 59: 611-618.

Bokemeyer M, Ding X-Q, Goldbecker A, Raab P, Heeren M, Arvanitis D et al (2011). Evidence for neuroinflammation and neuroprotection in HCV infection-associated encephalopathy. Gut 60: $370-377$.

Buchsbaum MS, Haier RJ, Potkin SG, Nuechterlein K, Bracha HS, Katz $M$ et al (1992). Frontostriatal disorder of cerebral metabolism in never-medicated schizophrenics. Arch Gen Psychiatry 49: 935-942.

Bush G, Luu P, Posner M (2000). Cognitive and emotional influences in anterior cingulate cortex. Trends Cogn Sci 4: 215-222.

Bustillo JR, Rowland LM, Lauriello J, Petropoulos H, Hammond R, Hart B et al (2002). High choline concentrations in the caudate nucleus in antipsychotic-naive patients with schizophrenia. Am J Psychiatry 159: 130-133.

Cao B, Passos IC, Mwangi B, Amaral-Silva H, Tannous J, Wu M-J et al (2017). Hippocampal subfield volumes in mood disorders. Mol Psychiatry (doi:10.1038/mp.2016.262).

Cao B, Passos IC, Mwangi B, Bauer IE, Zunta-Soares GB, Kapczinski F et al (2016a). Hippocampal volume and verbal memory performance in late-stage bipolar disorder. J Psychiatr Res 73: 102-107.

Cao B, Stanley JA, Selvaraj S, Mwangi B, Passos IC, Zunta-Soares G et al (2016b). Evidence of altered membrane phospholipid metabolism in the anterior cingulate cortex and striatum of patients with bipolar disorder I: a multi-voxel 1H MRS study. J Psychiatr Res 81: 48-55.

Chakraborty G, Mekala P, Yahya D, Wu G, Ledeen RW (2001). Intraneuronal $\mathrm{N}$-acetylaspartate supplies acetyl groups for myelin lipid synthesis: evidence for myelin-associated aspartoacylase. J Neurochem 78: 736-745.

Chang L, Munsaka SM, Kraft-Terry S, Ernst T (2013). Magnetic resonance spectroscopy to assess neuroinflammation and neuropathic pain. J Neuroimmune Pharmacol 8: 576-593.

Dager SR, Friedman SD, Parow A, Demopulos C, Stoll AL, Lyoo IK et al (2004). Brain metabolic alterations in medication-free patients with bipolar disorder. Arch Gen Psychiatry 61: 450-458.

Dale AM, Fischl B, Sereno MI (1999). Cortical surface-based analysis. I. Segmentation and surface reconstruction. Neuroimage 9: 179-194.

Fillman SG, Sinclair D, Fung SJ, Webster MJ, Shannon Weickert C (2014). Markers of inflammation and stress distinguish subsets of individuals with schizophrenia and bipolar disorder. Transl Psychiatry 4: e365.

Fountoulakis KN, Kontis D, Gonda X, Yatham LN (2013). A systematic review of the evidence on the treatment of rapid cycling bipolar disorder. Bipolar Disord 15: 115-137.

Frederick Bd, Satlin A, Wald LL, Hennen J, Bodick N, Renshaw PF (2002). Brain proton magnetic resonance spectroscopy in Alzheimer disease. Am J Geriatr Psychiatry 10: 81-88.

Gabbay V, Hess DA, Liu S, Babb JS, Klein RG, Gonen O (2007). Lateralized caudate metabolic abnormalities in adolescent major depressive disorder: a proton $\mathrm{mr}$ spectroscopy study. Am J Psychiatry 164: 1881-1889.

Grodd W, Krägeloh-Mann I, Petersen D, Karl Trefz F, Harzer K (1990). In vivo assessment of $\mathrm{N}$-acetylaspartate in brain in spongy degeneration (Canavan's disease) by proton spectroscopy. Lancet 336: 437-438.

Haase A, Frahm J, Hänicke W, Matthaei D (1985). 1H NMR chemical shift selective (CHESS) imaging. Phys Med Biol 30: 341-344.

Hamakawa H, Kato T, Murashita J, Kato N (1998). Quantitative proton magnetic resonance spectroscopy of the basal ganglia in patients with affective disorders. Eur Arch Psychiatry Clin Neurosci 248: 53-58.

Hamilton M (1980). Rating depressive patients. J Clin Psychiatry 41: $21-24$.

Janowsky D, Davis J, El-Yousef MK, Sekerke HJ (1972). A cholinergic-adrenergic hypothesis of mania and depression. Lancet 300: 632-635.

Kantarci K, Reynolds G, Petersen RC, Boeve BF, Knopman DS, Edland SD et al (2003). Proton MR spectroscopy in mild cognitive impairment and Alzheimer disease: comparison of 1.5 and $3 \mathrm{~T}$. AJNR Am J Neuroradiol 24: 843-849.

Kato T, Hamakawa H, Shioiri T, Murashita J, Takahashi Y, Takahashi S et al (1996). Choline-containing compounds detected 
by proton magnetic resonance spectroscopy in the basal ganglia in bipolar disorder. J Psychiatry Neurosci 21: 248-254.

Koukopoulos A, Sani G, Koukopoulos AE, Minnai GP, Girardi P, Pani L et al (2003). Duration and stability of the rapid-cycling course: a long-term personal follow-up of 109 patients. $J$ Affect Disord 73: 75-85.

Kreis R, Ernst T, Ross BD (1993). Absolute quantitation of water and metabolites in the human brain. II. Metabolite concentrations. J Magn Reson Ser B 102: 9-19.

Kupka RW, Luckenbaugh DA, Post RM, Suppes T, Altshuler LL, Keck PE et al (2005). Comparison of rapid-cycling and nonrapid-cycling bipolar disorder based on prospective mood ratings in 539 outpatients. Am J Psychiatry 162: 1273-1280.

Landim RCG, Edden RAE, Foerster B, Li LM, Covolan RJM, Castellano G (2016). Investigation of NAA and NAAG dynamics underlying visual stimulation using MEGA-PRESS in a functional MRS experiment. Magn Reson Imaging 34: 239-245.

Lavagnino L, Cao B, Mwangi B, Wu M-J, Sanches M, Zunta-Soares GB et al (2015). Changes in the corpus callosum in women with late-stage bipolar disorder. Acta Psychiatr Scand 131: 458-464.

Lee S, Tsang A, Kessler RC, Jin R, Sampson N, Andrade L et al (2010). Rapid-cycling bipolar disorder: cross-national community study. Br J Psychiatry 196: 217-225.

Mader I, Roser W, Kappos L, Hagberg G, Seelig J, Radue EW et al (2000). Serial proton MR spectroscopy of contrast-enhancing multiple sclerosis plaques: absolute metabolic values over 2 years during a clinical pharmacological study. Am J Neuroradiol 21: 1220-1227.

Mathers CD, Loncar D (2006). Projections of global mortality and burden of disease from 2002 to 2030. PLoS Med 3: 2011-2030.

McClure RJ, Kanfer JN, Panchalingam K, Klunk WE, Pettegrew JW (1994). Alzheimer's disease: membrane-associated metabolic changes. Ann NY Acad Sci 747: 110-124.

Michael N, Erfurth A, Pfleiderer B (2009). Elevated metabolites within dorsolateral prefrontal cortex in rapid cycling bipolar disorder. Psychiatry Res-Neuroimaging 172: 78-81.

Moore CM, Breeze JL, Gruber S A, Babb SM, Frederick BB, Villafuerte RA et al (2000). Choline, myo-inositol and mood in bipolar disorder: a proton magnetic resonance spectroscopic imaging study of the anterior cingulate cortex. Bipolar Disord 2: 207-216.

Mwangi B, Wu M-J, Cao B, Passos IC, Lavagnino L, Keser Z et al (2016). Individualized prediction and clinical staging of bipolar disorders using neuroanatomical biomarkers. Biol Psychiatry Cogn Neurosci Neuroimaging 1: 186-194.

Najjar S, Pearlman DM, Alper K, Najjar A, Devinsky O (2013). Neuroinflammation and psychiatric illness. J Neuroinflammation 10: 43.

Nordentoft M, Mortensen PB, Pedersen CB (2011). Absolute risk of suicide after first hospital contact in mental disorder. Arch Gen Psychiatry 68: 1058-1064.

Pettegrew JW, Keshavan M, Panchalingbam K, Strychor S, Kaplan DB, Tretta MG et al (1991). Alterations in brain highenergy phosphate and membrane phospholipid metabolism in first-episode, drug-naive schizophrenics. Arch Gen Psychiat 48: 563-568.

Phillips ML, Ladouceur CD, Drevets WC (2008). A neural model of voluntary and automatic emotion regulation: implications for understanding the pathophysiology and neurodevelopment of bipolar disorder. Mol Psychiatry 13: 829, 833-857.

Post RM, Denicoff KD, Leverich GS, Altshuler LL, Frye M A., Suppes TM et al (2003). Morbidity in 258 bipolar outpatients followed for 1 year with daily prospective ratings on the NIMH Life Chart Method. J Clin Psychiatry 64: 680-690.
Post RM, Fleming J, Kapczinski F (2012). Neurobiological correlates of illness progression in the recurrent affective disorders. J Psychiatr Res 46: 561-573.

Provencher SW (2001). Automatic quantitation of localized in vivo $1 \mathrm{H}$ spectra with LCModel. NMR Biomed 14: 260-264.

Rao JS, Harry GJ, Rapoport SI, Kim HW (2010). Increased excitotoxicity and neuroinflammatory markers in postmortem frontal cortex from bipolar disorder patients. Mol Psychiatry 15: 384-392.

Satlin A, Bodick N, Offen WW, Renshaw PF (1997). Brain proton magnetic resonance spectroscopy (1H-MRS) in Alzheimer's disease: changes after treatment with xanomeline, an M1 selective cholinergic agonist. Am J Psychiatry 154: 1459-1461.

Schubert F, Gallinat J, Seifert F, Rinneberg H (2004). Glutamate concentrations in human brain using single voxel proton magnetic resonance spectroscopy at 3 Tesla. Neuroimage 21: 1762-1771.

Seeger U, Klose U, Mader I, Grodd W, Nägele T (2003). Parameterized evaluation of macromolecules and lipids in proton MR spectroscopy of brain diseases. Magn Reson Med 49: 19-28.

Sesack SR, Grace AA (2010). Cortico-basal ganglia reward network: microcircuitry. Neuropsychopharmacology 35: 27-47.

Smith SM, Jenkinson M, Woolrich MW, Beckmann CF, Behrens TEJ, Johansen-Berg $\mathrm{H}$ et al (2004). Advances in functional and structural MR image analysis and implementation as FSL. Neuroimage 23: S208-S219.

Stanley JA (2002). In vivo magnetic resonance spectroscopy and its application to neuropsychiatric disorders. Can J Psychiatry 47: 315-326.

Stanley JA, Pettegrew JW, Keshavan MS (2000). Magnetic resonance spectroscopy in schizophrenia: methodological issues and findings-part I. Biol Psychiatry 48: 357-368.

Stanley JA, Vemulapalli M, Nutche J, Montrose DM, Sweeney JA, Pettegrew JW et al (2007). Reduced N-acetyl-aspartate levels in schizophrenia patients with a younger onset age: a single-voxel 1H spectroscopy study. Schizophr Res 93: 23-32.

Stertz L, Magalhães PVS, Kapczinski F (2013). Is bipolar disorder an inflammatory condition? The relevance of microglial activation. Curr Opin Psychiatry 26: 19-26.

Stork C, Renshaw PF (2005). Mitochondrial dysfunction in bipolar disorder: evidence from magnetic resonance spectroscopy research. Mol Psychiatry 10: 900-919.

Tamas G, Simon Andrea Lorincz A, Szabadics J (2003). Identified sources and targets of slow inhibition in the neocortex. Science (80-) 299: 1902-1905.

van Enkhuizen J, Janowsky DS, Olivier B, Minassian A, Perry W, Young JW et al (2014). The catecholaminergic-cholinergic balance hypothesis of bipolar disorder revisited. Eur J Pharmacol 753: 1-13.

Watkins CC, Sawa A, Pomper MG (2014). Glia and immune cell signaling in bipolar disorder: insights from neuropharmacology and molecular imaging to clinical application. Transl Psychiatry 4: e350.

Wu H, Goradia D, Stanley JA (2014). A Fully Automated and Robust Method of Extracting CSI voxels from Precise Anatomical Locations: An Application to a Longitudinal ${ }^{31} \mathrm{P}$ MRS Study. Proceedings of the 22nd Annual Meeting of International Society of Magnetic Resonance in Medicine. 10-16 May 2014. Milan, Italy. http://www.ismrm.org/

Young JW, Dulcis D (2015). Investigating the mechanism(s) underlying switching between states in bipolar disorder. Eur $J$ Pharmacol 759: 151-162.

Young RC, Biggs JT, Ziegler VE, Meyer DA (1978). A rating scale for mania: Reliability, validity and sensitivity. Br J Psychiatry 133: 429-435.

Supplementary Information accompanies the paper on the Neuropsychopharmacology website (http://www.nature.com/npp) 